\title{
LA INFORMÁTICA EDUCATIVA: UNA REFLEXIÓN CRÍTICA
}

\section{Álvaro Carvajal Villaplana ${ }^{1}$}

Resumen: Este artículo analiza la propuesta de la informática educativa optimista desde la perspectiva de la filosofía de la tecnología y las propuestas epistemológicas que la sustentan. El autor no se opone a la introducción de la computadora en el sistema educativo, pero sí cuestiona aquella perspectiva profética que considera que la inclusión de la computadora en el aula es la única vía para resolver el problema de la calidad de la educación.

Summary: The article analyzes the optimistic informatics educational proposal from the perspective of the philosophy of technology as well as from the epistemological ideas that support this proposal. The author does not oppose the introduction of computers in the educational system but questions the prophetic perspective that presents the use of computers in the classroom as the only means to solve the problem pertaining the quality of the education offered.

Palabras clave: INFORMÁTICA EDUCATIVA/ TECNOLOGÍA/ FILOSOFIA/ PROPUESTA EPISTEMOLÓGICA/ SISTEMA EDUCATIVO/ CALIDAD DE LA EDUCACIÓN/

\section{Introducción}

En la actualidad, se habla mucho contra el sistema de enseñanza tradicional, el cual, es catalogado de racionalista, formal y conductista. Sus principales defectos parten de una educación memorística, en la que el niño y el adolescente asumen una actitud pasiva ante el aprendizaje. El maestro se convierte en un transmisor de conocimiento, y el sistema, en el que funciona resulta autoritario, pues se orienta en una sola vía. Frente al sistema tradicional surgen nuevas propuestas teóricas y metodológicamente alternativas, que tienden a modificar la situación: escuela activa, constructivismo e informática educativa, entre otras. De esta variedad de posiciones, interesa aquí la informática educativa, especialmente la postura que sostiene sus fuentes epistemológicas en la inteligencia artificial (IA), la teoría cognoscitiva de Piaget y el constructivismo. La informática educativa no representa una posición conceptual homogénea; sin embargo, la tendencia a estudiar tiene su inspiración en Seymort Papert.

\footnotetext{
1 Profesor de las Escuelas de Estudios Generales y Filosofía de la Universidad de Costa Rica y de la Escuela de Ciencias Sociales del Instituto Tecnológico de Costa Rica. acarvaja@cariari.ucr.ac.ch
} 
En relación con dicha tendencia, se expondrán y analizarán algunos de los elementos teóricos y epistemológicos -sobre todo en relación con la propuesta de Papert-, así como sus críticas. También, se intentará precisar el término "informática educativa". Además, es de interés identificar el papel de las computadoras en el sistema educativo.

La informática educativa representa un reto para la educación, ya que las computadoras no pasan desapercibidas para los niños, pues ocupan el ambiente en que muchos de ellos se desenvuelven; por lo cual, de alguna forma, la educación tiene que incorporarla; no obstante, ese hecho no justifica una "visión triunfante" (Reparaz; 2000: 1), ni su consideración como propulsor determinante de un cambio radical del sistema educativo, pues se requieren otros elementos que la tecnología en sí no proporciona.

\section{Las visiones apocalíptica y "optimista ilustrada" de la tecnología}

Las actitudes que social e intelectualmente se asumen frente a la tecnología son de diversa índole; reconocerlas es conveniente para el análisis de cualquier tipo de tecnología, pues indican aspectos ideológicos e incluso míticos encontrados en las argumentaciones (a favor o en contra) en torno a ellas. También, sirven para comprender las orientaciones teóricas de quienes defienden determinadas posiciones.

En la novela filosófica de Robert Pirsig, Zen y el arte de la mantención de la motocicleta: una indagación sobre los valores (1974), se exponen dos formas de aproximación a la tecnología en la modernidad: (a) una de ellas se refiere a quienes tienen una relación armoniosa y positiva con la tecnología, lo cual se refleja en un interés por comprender los objetos y procesos tecnológicos; (b) la otra, tiene que ver con una aproximación de rechazo, resistencia o incomprensión de tales objetos y procesos. Esta distinción puede aplicarse al uso de las computadoras. Por una parte, algunas personas las asumen sin mayores dificultades, pero otras sienten un gran temor ante ellas. Tales actitudes, según Papert (1981, p. 20-21), son influenciadas por la cultura: algunas favorecen más las actitudes positivas, mientras que otras promueven las negativas. El enfoque de la informática educativa de Papert pretende modificar la disposición negativa, suscitando una actitud positiva en los niños. 
Por su parte, Carl Mitchan (1989) considera que, ante la tecnología, existen dos maneras de reaccionar: (a) la primera es el escepticismo tecnológico, el cual consiste en ver la tecnología como un mal inevitable: si bien la tecnología puede ayudar en la vida cotidiana, aleja al ser humano de lo natural. Esta es una postura asumida por ciertos grupos religiosos, como los talibanes, melamitas, amish, testigos de Jehová, entre otros, que rechazan algún tipo de tecnología. (b) La otra es el optimismo ilustrado, en el cual se ubica a Papert Negroponte (1995, p. 21) y sus seguidores. Para Mitchan esta posición asume que la tecnología posee una bondad inherente y su mal es accidental. En caso extremo, el optimismo ilustrado coloca ante todo a la tecnología y la modernización como lo prioritario; así, el conocimiento adquirido por un contacto técnico con el mundo es más auténtico que la teoría abstracta. Esto es continuamente expuesto por Papert: las computadoras son un instrumento adecuado de aprendizaje, pues ponen al niño en contacto con lo real, incluso le crean ambientes de conocimiento a los cuales no podría tener acceso de manera natural. En este autor, así como en Negroponte, la reflexión ética sobre la tecnología o el uso de la computadoras por parte de los niños está ausente, pues asumen que esta tecnología es en sí misma buena o, en el caso moderado, neutra $\mathbf{0}$

En relación con las actitudes del optimismo y pesimismo tecnológicos en conexión con el tema de la informática educativa, es importante rescatar algunas ideas de Luis Camacho (1993: 181), quien apunta una distinción, propuesta por Lewis Munford, en Técnica y civilización, entre dos tipos de tecnología: (a) las capacitadoras y (b) las definitorias. Las primeras contribuyen a ejecutar con mayor eficiencia las tareas cotidianas; las segundas, tienen un impacto profundo en la sociedad, llegando a determinar cuáles son las tareas diarias, así como a definir la clase de sociedad en la que viven los individuos que la utilizan.

\footnotetext{
${ }^{2}$ Aunque, Papert recientemente, no se considera un optimista extremo (1997: 33).

${ }^{3}$ Respecto al último punto, cabe destacar la posición de Jaime Sánchez, para quien la computadora es un instrumento neutral, para él es "el hombre el que lo utiliza para multiplicar el efecto de una acción, reproducirla, ampliarla o reducirla. El computador en sí no es bueno ni malo: los efectos que produzca serán o no beneficiosos, según cómo el hombre use el computador" (Sánchez, 1995: 123). El problema es que la idea de la neutralidad de la tecnología sólo sería correcta en un mundo en donde la tecnología nunca está separada de los valores, de las estructuras de poder, de la dirección predeterminada de la investigación por aquellos que la financian, en fin, en donde no existiesen seres humanos. La idea de la neutralidad tecnológica no es nueva. Desconoce además que hay tecnologías, como lo sugiere Roy Ramírez (1995), que en sí mismas son perniciosas, destructoras del ambiente, promotoras de la guerra y de la dominación del ser humano; por ejemplo, los agroquímicos o la bomba atómica, cuya única finalidad es la guerra
} 
El ejemplo de Munford es la sociedad medieval benedictina, cuyos conventos funcionaron como si fueran un reloj. La dificultad estriba en clasificar, en una u otra categoría, a las diferentes tecnologías. Para Camacho (1983), la tecnología de la computación es definitoria, ya que aparte de simplificar las labores cotidianas, influye enormemente en la vida humana, llegando a cambiar la imagen que el ser humano tiene de sí mismo y de la sociedad en la que vive, así como lo que se considera deseable en ella. Esto en razón de los criterios aportados por el modelo de Fridman (1974, p. 53-54) sobre la manera en que la tecnología afecta, influye y modifica los valores de una sociedad

Según Camacho, la idea de Munford según la cual las sociedades se organizan conforme al tipo de tecnología que utilizan, implica que la sociedad es una gigantesca máquina. Esta metáfora llevada a su extremo conduciría a la idea de que los seres humanos se comportan como máquinas y no habría diferencias entre ambos (Camacho, 1993, p. 182). En la informática educativa tal idea se manifiesta en expresiones como "la computadora es una extensión del cerebro humano", o en el caso de la inteligencia artificial, cuando se análoga la computadora con la mente humana, mucho más irónica es la semejanza establecida en expresiones como: el niño se comunica con la máquinal o cuando se habla de una mente (la del niño) que se comunica con otra mente (la de la computadora), estas metáforas dan por supuesto que la máquina tiene características semejantes a las que tiene la mente humana.

La idea de la tecnología como extensión del cuerpo humano, se expresa con claridad en la postura de Jaime Sánchez, cuando afirma que "el hombre comienza a sentir el poder de la máquina como la prolongación de su propio yo" (Sánchez, 1995, p. 11), dicha prolongación se considera como un paso evolutivo de la especie humana. Referencia semejante se halla en Dianelos Georgoudis (citado por Rifkin, 1994, p. 113), quien piensa que el libro es una extensión de la mente del autor, por analogía, la computadora es una extensión de la mente humana. Dicha idea recuerda la concepción de la tecnología de Ernest Kapp, quien en el siglo XIX la entiende como una proyección de los órganos (citado por Mitchan: 1989, p. 25-29). Según Mitchan, esto se enmarca en un enfoque de la

\footnotetext{
${ }^{4}$ Sobre cómo la tecnología modifica los valores y la sociedad, también consúltese a Jean Ladrière, El reto de la racionalidad (1974).

${ }^{5}$ Obviamente, entre la computadora y el niño existe algún tipo de comunicación rudimentaria, pero esta comunicación es sintáctica mas no comprensiva.
} 
tecnología que él denomina ingeniería mecanicista. Enfoque concordante con una de las fuentes de la informática educativa, aquella que tiene su origen en las concepciones mecanicistas ingenieriles y del aprendizaje.

Según Camacho, cuando una tecnología definitoria comienza a difundirse y a modificar los hábitos y los valores de las personas, aparecen los profetas mesiánicos y los apocalípticos; esto en correspondencia con el optimismo y el pesimismo tecnológico. Distinción común a la literatura de la segunda parte del siglo XX. Mientras que "los primeros anuncian la llegada del Mesías que resolverá los problemas de la humanidad, la nueva tecnología es entonces saludada como la solución a las miserias, hambres, conflictos y congojas del ser humano. El profeta apocalíptico, en cambio, vaticina terribles calamidades conectadas con los nuevos aparatos" (Camacho, 1993, p. 183). El contraste se aplica a la informática educativa, siendo Papert un representante de lo primero, aunque es especialmente notable cómo sus ideas influyen en el pensamiento de otros estudiosos, como Carlos Devandas, quien asevera que los aportes de Papert

han variado la idea misma del cambio social, de las sociedades futuras, han abierto nuevas formas de entender la solidaridad entre hombres y pueblos $\mathrm{y}$, decididamente, a partir de un fondo humanista que cala muy hondo nos ha abierto a los pueblos latinoamericanos y del Tercer Mundo en general, un camino de esperanza cuyo recorrido no depende de las armas, ni la intriga o los odios, sino del trabajo conjunto, del esfuerzo intelectual y de la voluntad por ser mejores y construir un mundo mejor para todos. (Devandas, 1985, p. 131).

Las actitudes mesiánicas o apocalípticas se aprecian en las maneras de considerar las repercusiones de la tecnología informática en el ámbito laboral. Para los defensores, como Jaime Sánchez, la computadora permitirá a:

las grandes empresas que hoy desarrollan sus tareas en enormes plantas y edificios poblados de empleados, tal vez, en un futuro cercano, resolver este problema teniendo una planta más pequeña, con mucho menos gente, donde habrá un computador central y cada empleado tendrá en su casa un microcomputador o una terminal, y el inicio de su trabajo se limitará a enchufar y encender su microcomputador y leer en pantalla las instrucciones entregadas por la central, respondiendo e interactuando con ella. (Sánchez, 1995, p. 13). 
La visión apocalíptica de Jeremy Rifkin expuesta en El fin del trabajo, presagia cómo la tecnología informática desplazará por completo al ser humano limitándole al mínimo sus opciones laborales (Rifkin, 1994). Dicho punto de vista coincide con el optimismo de Georgoudis, para quien la computadora podría sustituir en un $90 \%$ las tareas del profesor (1985: 118) Mientras que la primera opción ve en el desplazamiento de los seres humanos por la máquina una oportunidad para que éstos desarrollen sus capacidades creativas e imaginarias, lo anterior, suponiendo que no tendrán que angustiase por la búsqueda del sustento diario. Por el contrario, posiciones como la de Rifkin son alarmantes, pues si bien las personas no tendrán que realizar los trabajos tediosos, alienantes e insalubres, tampoco poseerán un empleo o los ingresos suficientes que aseguren los niveles mínimos de supervivencia. En el ámbito de la informática educativa, mientras que para algunos optimistas el educador ocupará un lugar importante como estimulador de la creatividad, el pensamiento crítico y lógico de los estudiantes, en el enfoque de Rifkin, el maestro queda desplazado por la máquina.

Para algunos defensores de la informática educativa, es característico acusar de dogmáticos (por ejemplo, Sánchez) a los críticos del uso de ese tipo tecnología. Sin embargo, habría que preguntarse quiénes son los dogmáticos, si los que asumen una posición crítica frente a las nuevas tecnologías o aquellos que adoptan una actitud de complacencia o aceptación de determinada tecnología. Por otra parte, en general, entre los defensores de la informática educativa, sólo se encuentran halagos, mas no análisis de los problemas que trae consigo. Cabe advertir que el estudio de la informática educativa a la que en este artículo se hace referencia, sólo comprende aquellas posturas extremas sumamente optimistas. No obstante, dada la variedad de posiciones, es importante pasar a definir lo que se entiende por informática educativa.

\section{Caracterización de la informática educativa}

El concepto de "informática educativa" no es uniforme. Existen diferentes maneras de concebirlo, las cuales van desde las nociones más restringidas hasta las más amplias y flexibles. Deben distinguirse y valorarse las expresiones: cultura e informática educativa. La cultura informática se refiere a la creación de una actitud que permita integrar la

\footnotetext{
${ }^{6}$ Pero, según Raparaz, las posturas de la informática educativa que pretendían la sustitución del profesor por la computadora se han abandonado (2000, p. 13).
} 
computadora a la vida cotidiana de los seres humanos. Esto por cuanto se considera que la computadora y la informática llegarán a ser las herramientas más comunes dentro de la sociedad. La importancia que se concede a esta máquina, en tanto tecnología definitoria del nuevo tipo de sociedad, fue resaltado en enero de 1983 en una edición especial de la revista Time titulada El hombre del año, en donde el "hombre del año" se reemplazó por el titular La máquina del año, haciendo mención al computador como la herramienta más popular en 1982.

La preeminencia tanto de la computadora como de la informática, se debe, entre otros aspectos, a la idea de que el logro del desarrollo en un mercado globalizado pasa por el dominio de dicha tecnología. Las sociedades que no asuman una actitud positiva frente a ella, están condenadas al subdesarrollo. Desde esa misma óptica y como consecuencia de la globalización, la competencia aparece como un elemento del cual no pueden escapar las sociedades; la optimización de las economías transita por el manejo y producción de tecnologías que aporten mayor valor agregado a los productos y a los recursos humanos. De manera que la informática es una tecnología con la que se logra optimización, eficiencia y productividad. Tal idea es frecuente en la justificación de los libros o artículos que defienden la incorporan de la computadora y la informática al currículo escolar.

El concepto de cultura informática es de difícil definición, pues no se precisa su significado. Una aproximación la define como el "desarrollo de un ciudadano informado, capaz de entender el impacto social y político de la informática y en especial de las computadoras en la sociedad, así como un entendimiento general de las características y operaciones del computador (Sánchez, 1995, p. 18). ¿Cómo entender esta definición tan amplia? A dicha pregunta se le pueden dar tres respuestas: la primera, referida a una acepción amplia del término, el cual comprende varios aspectos: (a) la creación de una conciencia sobre la presencia de la computación en la sociedad actual que permita el desarrollo de actitudes positivas hacia esta tecnología; (b) el conocimiento y análisis del impacto de la computación y la informática en la sociedad; (c) una mejora cultural de todas las personas que facilite la seguridad y la toma de decisiones, lo cual evitaría que los usuarios fueran simples manipuladores o técnicos que sólo poseen nociones y entendimiento teórico fragmentado sobre los componentes y el funcionamiento del computador. 
La segunda respuesta identifica "cultura informática" con programación computacional. Un individuo desarrollado es aquel que posee un lenguaje artificial que facilita la comunicación con la computadora (Papert, 1981, p. 18). La persona debe poseer una serie de elementos informáticos que conformarían parte integral de su conocimiento básico. En contraposición, puede argumentarse que no parece indispensable que todas las personas tengan que saber programar, pues ésta es una tarea muy especializada. Por otra parte, la cultura informática como sinónimo de programación, proviene de especialistas que se sienten muy comprometidos con la inteligencia artificial y que suponen que una persona educada es aquella que sabe programar.

Una tercera postura procura la mediación entre las anteriores. No obstante, por razones de espacio no es posible exponerlo aquí (Cabero: 2001; Reparaz: 2000). En todo caso, las acepciones de "cultura informática" remiten al concepto de "alfabetización informática", término sobre el cual tampoco existe consenso en torno a su significado.

La denominada "informática educativa" es un término técnico que se refiere de manera precisa a la introducción de la informática al currículo escolar. Las razones para su inclusión y las formas de hacerlo son múltiples. Existen varias formas de concebir dichos usos (Cabero, 2001 Seas y otros, 1999; Georgoudis, 1985). Una de las más completas es la presentada por Sánchez, quien distingue cinco enfoques: aprendizaje acerca de la computadora (alfabetización), aprendizaje por medio del computador (programas de ejercitación y tutorial); aprendizaje con el computador (herramienta instruccional); aprendizaje acerca del "pensamiento" del computador (una herramienta para pensar, esta es la óptica de Papert) y administración del aprendizaje con el computador (1995, p. 91-93).

Las anteriores formas de introducir la informática a la educación son indicadoras de la no exclusividad de un uso determinado de dicha herramienta de aprendizaje, como pretenden aquellos que defienden posiciones constructivistas o quienes dan mucha importancia a la programación, es decir, la computación como un instrumento de aprendizaje o el aprendizaje computacional o informático como un fin en sí mismo. Por ejemplo, para Papert, a pesar de reconocer -en algún momento- que el uso de la tecnología es una más entre otras, en el fondo considera que la computadora no es tan solo un instrumento de apoyo cualquiera, si no que se trata del instrumento por excelencia, al parecer, la única vía posible para aprender en el futuro. 
Dicha tecnología, como bien lo afirma Cabero (2001, p. 308-309), está en función de los fines educativos y los objetivos curriculares. Se encuentra al servicio de la labor educativa y no a la inversa. Por otra parte, es importante entender que existen maneras diversas mediante las cuales las personas se apoderan de esta herramienta, igualmente no puede imponerse un sólo estilo de aprendizaje. Para algunos, la habilidad de programar será indispensable, para otros será el utilizar la máquina, emplear los programas, las bases de datos y el procesador de texto. Tampoco, la presencia de la computadora en la escuela es suficiente para despertar el interés en el niño hacia ellas, ni para provocar emociones fuertes en éstos hacia las materias que se enseñan con ellas.

Para concluir este apartado, es importante destacar que son escasas las investigaciones realizadas para determinar el impacto de la inclusión de las computadoras en el currículo para el mejoramiento del aprendizaje. Por lo general, las opiniones favorables están basadas en anécdotas de situaciones de casos especiales y exitosos, por ejemplo, las situaciones que relata Papert. Además, el uso de esta tecnología es afortunada para las personas con discapacidad y problemas de aprendizaje. Empero, no existen datos confiables que muestren que dicho "éxito" pueda generalizarse a toda la población escolar

\section{Supuestos epistemológicos de la informática educativa}

En el apartado 3 se presentó una gama de enfoques sobre la informática educativa, todos pertinentes a los procesos de enseñanza en tanto que la computadora sea vista como una herramienta más de aprendizaje en función del currículo. En la práctica, los diferentes modelos oficiales de incorporación de tal tecnología a la escuela, no tienden a modificar de manera total el sistema tradicional educativo, lo que puede interpretarse como un refuerzo de la idea de que la informática educativa es una alternativa más dentro del amplio abanico de tecnologías educativas. Por otra parte, las posiciones radicales opinan que el sistema educativo debe modificarse drásticamente; según Papert, tiene que darse un megacambio que sólo es posible por medio de la computadora. Tanto las posiciones moderadas como las extremas presentan unas fuentes teóricas que les son comunes, mucha de la cual fue aportada por Papert.. Aquí interesa incursionar en los fundamentos de las tendencias o enfoques disciplinares que dan origen o están a la base del tema de estudio de este artículo,

\footnotetext{
${ }^{7}$ Véase por ejemplo las críticas de Zayra Méndez (1987: 161-166) y Rafael Ángel Herra (1987, p. 181 -185), entre otros. Un fuerte crítica a la propuesta de programación por medio del lenguaje logo de Papert se encuentra en José Chelque (1999, p. 184-187).
} 
ellas son: la inteligencia artificial, el cognoscitivismo de Piaget y el construccionismo de Papert.

La confluencia de estas tres tendencias en la teoría de Papert contribuye a la formación de la visión optimista ilustrada de las primeras décadas de la informática educativa.

\subsection{La inteligencia artificial (I.A.)}

Existen dos maneras de concebir la mente, y por ende la inteligencia. Una es el enfoque biológico y la otra, el artificial o inteligencia artificial. El primero se refiere a un sistema físico, orgánico y vivo caracterizado por determinado tipo de células: las neuronas. El segundo, considera la inteligencia como una propiedad formal de cualquier sistema que reúna ciertos requisitos funcionales, es el caso particular de una máquina, la cual tiene un soporte físico pero inorgánico. Al primer enfoque, le resulta irritante la pregunta de si las máquinas piensan; en cambio, para el otro, esa pregunta no es necesario planteársela, pues se da por hecho que es posible la inteligencia inorgánica.

Por otro lado, John Searle distingue dos maneras de concebir la IA; uno fuerte y otro débil (Searle, 1984, p. 33). El sentido fuerte se expresa en la metáfora "la mente es al cerebro lo que el programa es al hardware del computador", es decir, se establece una analogía entre la mente y la computadora. Al mismo tiempo, esta perspectiva sostiene que las operaciones de la computadora -en los programas más avanzados- son equivalentes a las operaciones mentales humanas. Según lo anterior si las máquinas son capaces de responder preguntas, entonces son inteligentes, y esta actividad de dar respuesta explica lo que hace la mente humana. En cambio, la IA débil considera que la computadora sólo es útil para entender el funcionamiento del cerebro humano, sin afirmar la equivalencia entre los estados mentales y las operaciones de la máquina. La crítica de Searle se dirige a la IA fuerte.

\footnotetext{
${ }^{8}$ En general, en la literatura sobre informática educativa, la mayoría de los autores se refiere a la inteligencia artificial como una fuente teórica de dicha tendencia pedagógica, pero al parecer, este es un recurso ideológico que refuerza la idea del opitimismo ilustrado, porque pareciera que la preocupación fundamental se reduce a la cuestión de si el niño o el adolescente deben aprender a programar.
} 
En su crítica a la ciencia cognitiva que sustenta la Al fuerte, Searle establece cuatro rasgos de los fenómenos mentales que cualquier explicación satisfactoria de la mente debiera considerar: (a) la existencia y funcionamiento de la conciencia; (b) la intencionalidad, mediante la cual se dirigen los estados mentales, (c) la subjetividad de los estados mentales y (d) la causación mental (Searle, 1984, p. 20-21). Estos principios deben tomarse en cuenta para evaluar la creación de inteligencia artificial. Hasta la fecha, los programas de computación no reproducen tales rasgos. El principio general de evaluación reside en que la "manera de probar cualquier teoría de la mente consiste en preguntarse qué ocurriría si la mente de uno funcionase según los principios que, de acuerdo con la teoría, rigen todas las mentes" (Camacho, 1985: 190). Para ello recurre al ejemplo del cuarto chino. Este experimento mental aporta una imagen clara de la diferencia entre comprender y operar sin entender, aunque no sepamos aun en qué consiste comprender. Para Searle es importante establecer la distinción entre las operaciones mentales de entender algo y la de manipular signos correctamente, por medio de la sintaxis pero sin comprender (Searle, 1984: 45-46). La objeción tiene que ver con que no puede darse inteligencia como la humana sin la existencia de la intencionalidad y la consciencia; por tanto, no puede hablarse de IA en sentido estricto si no se resuelve el problema de la intencionalidad y la consciencia.

Por su parte, Papert distingue en Desafío a la mente entre la IA fuerte y la débil; pero no rechaza el supuesto principal de la primera, en sus palabras:

la definición de inteligencia artificial puede ser estricta o amplia. En sentido estricto, la IA se ocupa de ampliar la capacidad de las máquinas para realizar funciones que se considerarían inteligentes si las realizaran personas. Su objetivo es construir máquinas (...). Para construirlas genialmente, es necesario reflexionar no solamente sobre la naturaleza de las máquinas sino también sobre la naturaleza de las funciones inteligentes que deben ser realizadas (...) a fin de hacer una máquina capaz de aprender, tenemos que sondear profundamente la naturaleza del aprendizaje. $Y$ este tipo de investigación surge la definición más amplia de inteligencia artificial: la de una ciencia cognoscitiva. En este sentido lo propio de la IA es que su metodología y su estilos de teorización remiten marcadamente a teorías de computación (Papert, 1981, p. 182).

En la inteligencia artificial se usan modelos computacionales para obtener una percepción profunda de la psicología humana, también para reflexionar sobre ésta como Volumen 2, Número 1, Año 200211 
fuente de ideas para generar mecanismos que emulen la inteligencia humana. Para él "esta empresa impresiona a muchos como algo ilógico; aun cuando el desempeño parezca idéntico, ¿hay alguna razón para pensar que los procesos subyacentes no son los mismos?" (Papert, 1981, p. 190). Papert no encuentra ninguna, y afirma que si en la actualidad la IA no ha logrado sus objetivos no hay ningún impedimento para alcanzarlos, y para ello utiliza la analogía con la aparición de la primera máquina de volar. Incluso, un optimista ilustrado como Minsky afirma que "dentro de 50 años los ordenadores tendrán emociones" (Minsky, 2000, p. 20).

Si bien en Papert la argumentación inicial es que las computadoras son útiles para entender los procesos mentales y de aprendizaje, en el fondo, sostiene la premisa de que las computadoras son inteligentes. Hay que esperar a la aparición de La maquina de los niños para que Papert asevere que él nunca pensó que la IA estuviera simulando realmente la conducta humana y que lo único que le interesaba era la metodología (Papert, 1994: 182). No obstante, persiste en la idea de que el niño pueda construir programas inteligentes que simulen el orden biológico (1994, p. 196).च

La manera como Papert relaciona la IA con la teoría del aprendizaje de Piaget es interesante. Según Papert, a la IA le interesa dar formas concretas a ideas abstractas incluso metafísicas; es esa cualidad concretizadora de la IA lo que le resulta atractivo. De aquí desprende Papert que los niños pueden usar ideas de manera más informal y personal para pensar sobre sí mismos (Papert; 1981, p. 183). Lo que no es claro es si los niños logran llevar a cabo este fin de manera generalizada.

Según Sánchez, desde el punto de vista educativo, se pretende que la IA module al profesor, al estudiante y al medio ambiente educativo, contribuyendo así al entendimiento de los principios básicos e indescifrables de la inteligencia, razonamiento y conocimiento. La máquina supera al ser humano en cuanto a velocidad, eficiencia y confiabilidad en el procesamiento de información, y compite con éste en algunas áreas intelectuales. A los que se oponen a esta visión los llama pesimistas, negativos y dogmáticos, y a los que la definen,

\footnotetext{
${ }^{9}$ El tema de la inteligencia artificial es mucho más complejo de lo aquí se presenta, encontrándose diferentes niveles de investigación e intervención tecnológica que no son equivalentes, pero que en la literatura de la informática educativa tienen a confundirse, ellos son: (1) la IA como herramienta técnica; (2) la IA como investigación práctica en inteligencia; (3) la IA como instrumento de modelización, entre otros. No obstante, en este artículo no es posible clarificar estos diferentes aspectos.
} 
optimistas. Él se considera un optimista, aunque suaviza su posición diciendo que la analogía no es estricta, no se trata de que el ser humano sea como una máquina, pues aparte de ser razón y lógica, también es intuición, sensualidad, emoción, y sentimiento. Las máquinas sólo se aproximan a las dos primeras; pero entonces, ¿es la mente una máquina? No obstante, su justificación optimista de la informática educativa le conduce a pensar que para el año 2000 la inteligencia artificial estará tan desarrollada que las máquinas podrán razonar, aprender y planificar en formas que los humanos consideran inteligentes (1995, p.15). Como es obvio su predicción no se cumplió. Para él IA "intenta que las máquinas razonen como sus inventores; que traduzcan lenguajes, que respondan a la voz humana; que reconozcan imágenes" (1995, p. 63); y que muestren rasgos de conciencia, voluntad, y creatividad, en suma que sean capaces de desarrollar una conducta autodeterminada, intencional y de acuerdo a mentes.

Hechas las anteriores acotaciones puede retomarse la relación hecha por Sánchez entre la IA y Piaget. Para él, la Al "estudia mentes para producir programas y usa los programas para pensar acerca de la mente. La inteligencia artificial entiende la mente como una construcción de programas. Proyecta el constructivismo a su manera. Es el hombre el que construye sus mentes, sus máquinas. Mentes construyen mentes" (Sánchez, 1995, p.70). Los programas pueden ser construidos de manera que utilicen reglas heurísticas o empíricas que representen suposiciones inteligentes de cómo proceder para resolver el problema. Pueden analizar las alternativas y formular nuevas reglas heurísticas, creando así elementos desde los cuales emerge una nueva inteligencia no generada ni anticipada por el programador. Como se ve, es difícil distinguir cuándo los proponentes de la IA se refieren a la débil y cuando a la fuerte. La segunda propuesta, la IA débil, es muy ambigua, por lo general, quienes la sostienen terminan sustentando los supuestos de la IA fuerte.

La crítica puede ser enfocada desde otra perspectiva, según Juan Ignacio Pozo las teorías cognoscitivistas que tienen como base el modelo constructivista, en última instancia conciben la analogía entre máquina y mente como procesadores de información. Así, según él, se adoptan los programas de computadora como metáfora del funcionamiento cognitivo humano. Este tipo de teorías afirman que el proceso o evolución cognitiva sólo puede ser comprendido reduciéndolo a las unidades mínimas de que está compuesto (Pozo, 1989, p.44). Estos componentes se unen entre sí para formar un programa por medio de reglas sintácticas. Los modelos cognitivos por información son por tanto lineales y aditivos. Sin Volumen 2, Número 1, Año 200213 
embargo, el cerebro humano no es ni lineal ni aditivo, sino paralelo, la IA todavía no ha podido emular este tipo de razonamiento, aunque existen intentos en la llamada cibernética. En este sentido, a pesar del pretendido ataque, por ejemplo de Papert, al énfasis que la sociedad occidental da a la lógica y a la formalización, estas teorías conciben la computadora como sistemas lógicos o matemáticos de procesamiento de información, constituidos exclusivamente como procedimientos formales. En este sentido, dichas teorías no se diferencian del conductismo.

Desde el punto de vista de la IA y las ciencias cognitivas, los elementos afectivos, los aspectos de la conciencia y la cultura en su inicio no tuvieron relevancia. Esos elementos fueron proporcionados por otro ámbito de teorías; en este caso, el cognotivismo de Piaget. La computadora, entonces, no emula en su totalidad la mente humana, sino sólo una parte de ella, la que tiene que ver con los aspectos sintácticos, formales, matemáticos y lógicos; aún así, es dudoso que haya comprensión. Aparentemente, la posición de Papert en El desafío de la mente, es del tipo aquí descrita, mientras que en La máquina de los niños, parece darse cuenta de lo limitado de su programa Logo, el cual cambia por el enfoque cibernético. Este nuevo enfoque es un intento por desarrollar en las máquinas memoria semántica y no tanto sintáctica; es decir, racionamiento en paralelo, de tal forma que "la memoria semántica está constituida por redes asociativas" (Pozo; 1989, p. 49). Dicho cambio es tan sólo de un incremento cuantitativo, aún no logra emular la parte semántica.

La analogía de la mente con el computador tiene un problema fundamental, pues como lo expuso Searle, lo que manipula la computadora es información y no significados; la información se mide en términos de probabilidad matemática. Por tanto, ¿de qué tipo de comunicación se habla cuando se dice que el "niño se comunica con la máquina"'?, acaso, ¿entiende la máquina lo que él niño le dice? Esta fuente teórica de la informática educativa es mecanicista. La cuestión es saber cómo puede armonizarse la perspectiva mecánica con el organicismo de Piaget. Este es un dilema que no resuelve Papert, ya que él se limita a hacer una llana combinación de elementos de ambas teorías.

\subsection{El cognoscitivismo de Piaget}

En general, las teorías cognitivas, especialmente las tendencias europeas, describen el aprendizaje como construcción, modificación, organización, estructuración, reestructuración 
y readecuación de estructuras mentales. El aprendizaje es visto como un cambio en el significado de las experiencias del alumno, de manera que éste pueda construir nuevos y poderosos significados. En lugar de visualizar el aprendizaje como una modificación de la conducta, se habla de una modificación en el aprendiz. Así un contenido se aprende cuando es adquirido, procesado, retenido y recuperado flexiblemente. Aunque estas teorías no son uniformes y presentan diferencias, tienen en común los rasgos descritos anteriormente.

En Piaget, se distinguen dos tipos de aprendizaje: uno en sentido estricto, que se adquiere del medio de información específico. El segundo tipo es la acepción amplia de aprendizaje, la cual consiste en el progreso de las estructuras cognitivas por medio de los procesos de equilibración, lo que remite a los estadios de evolución del conocimiento. Piaget rechaza el aprendizaje por asociación de tipo conductista. Para él, lo importante es explicar cómo procede el sujeto para construir e inventar, pues las personas simplemente no repiten ni copian. Según su teoría del equilibrio del aprendizaje, éste último se produce cuando hay un desequilibrio o un conflicto cognitivo.

Un elemento importante de esta teoría es la asimilación (Piaget, 1970, p. 17), es decir, la integración de los nuevos elementos que son externos a las estructuras en evolución; es decir, la interpretación de la información que proviene del medio, en función de sus estructuras conceptuales disponibles. En consecuencia, este tipo de teorías tienen como base algún tipo de Gestalt. Es el sujeto el que da el significado al mundo, no es éste último el que tiene significado, como pretende el conductivismo, ¿se trata a caso de una forma de idealismo? El proceso complementario es la acomodación, gracias al cual los conceptos e ideas se adaptan recíprocamente a las características reales del mundo, si no fuese de esta manera, los seres humanos vivirían en un lugar de fantasía. Esto tiene que ver con la diferencia entre ver y mirar. Aspectos que Kuhn utiliza y aclara en la Postdata a la Estructura de las revoluciones científicas.

El progreso de las estructuras cognitivas se produce por un equilibrio entre los procesos de asimilación y acomodación, (Piaget, 1970, p. 18), sobre los cuales no es posible profundizar en este artículo. Hay dos tipos de respuestas a las perturbaciones o estados de desequilibrio: las no adaptativas, en las que el individuo no toma conciencia de ellas, y en donde el sujeto no concibe la perturbación como conflictiva, por tanto, no intenta una 
modificación de las estructuras, y las adaptativas, en las que el sujeto toma conciencia de la perturbación e intenta resolverlas.

La crítica de Pozo a la teoría de Piaget estriba en que existen pruebas empíricas en contra de la noción piagetiana del aprendizaje como un sucedáneo del desarrollo (Cfr. Chomsky y Karmiloff-Smith, quienes hacen críticas más agudas a Piaget). Por otro lado, están los problemas teóricos del modelo de aprendizaje por equilibrio. El primer tipo no proporciona conclusiones definitivas, por lo que no interesa desarrollarla aquí. El segundo, es más interesante, por cuanto es una crítica aplicable a la propuesta de Papert, por cuanto él asume a Piaget con escasas modificaciones. Para Pozo "uno de los más graves problemas a que se enfrenta la teoría piagetiana de la equilibración es que supuestamente debe explicar la aparición de estructuras generales de conocimiento que poseen un carácter necesario o universal" (Pozo; 1989, p. 189). Ahora, ¿cómo esas estructuras pueden ser necesarias sin ser innatas? Esto conduce a una paradoja de la teoría: cada persona construye individualmente su propio conocimiento, pero al final, todas las personas acaban concluyendo el mismo conocimiento, es decir, hay una idea de finalismo en la teoría (Pozo, 1989: 190). Lo cual también es perseguido por las teorías tradicionales racionalistas. Tal parece, que los datos muestran que la adquisición de muchos conocimientos formales no se producen en el estadio formal, sino que según Pozo, "los desequilibrios que proceden de la reestructuración de los conocimientos se producen en el seno de estructuras de conocimientos específicos, de forma que lo que el sujeto aprenderá finalmente no será una estructura general sino un conjunto de conocimientos específicos que le harán ser más o menos experto en un estadio de conocimiento, pero que no necesariamente implicarán un progreso a un estadio cognitivo superior" (Pozo, 1989, p. 190); esta es la paradoja del aprendizaje.

El cognotivismo de Piaget no explica cuándo se resuelven favorablemente los desequilibrios, pues "el aprendizaje se concibe como post-efecto de la aplicación de una estructura o, dicho de otra forma la adquisición de conocimientos específicos es un efecto de la reorganización de las estructuras cognitivas generales y no al revés" (Pozo, 1989, p. 190). Por eso, al negar el aprendizaje por acumulación, se cae en la paradoja del aprendizaje, pues se postulan estructuras necesarias pero no predeterminadas. 
La salida a este conflicto lo ve Pozo en aquellas teorías que puedan relacionar el aprendizaje asociativo con la reestructuración, esta posición es la que puede hallarse en Vygotsky. Una línea de reflexión semejante lleva a otros autores a plantear la necesidad de crear un híbrido por medio del establecimiento de relaciones entre el sistema educativo tradicional, es decir, el aprendizaje por acumulación o método racionalista, con el constructivista, este es el caso de Eduardo Saxe (1998). También, del artículo de Luzia Marta Bellini (1998), se desprende esa misma paradoja, aunque su planteamiento es más modesto y práctico; lo que ella constata es que los dos modelos teóricos (el tradicional y el constructivista) sobreviven paralelamente, y que el niño se ve enfrentado a ambos, aunque ella opta preferencialmente por el modelo de Papert.

\subsection{El constructivismo de Papert}

Papert adopta muchos supuestos teóricos de la IA y de la epistemología genética de Piaget. En lo que sigue, interesa destacar algunos aspectos relevantes de la propuesta de Papert. Mientras que en el empirismo, el aprendizaje se da por factores externos, en Piaget, es esencialmente interno y se activa con la experiencia externa, idea que recuerda a Kant. El mismo Papert explica esta tendencia cuando afirma que las estructuras de conocimiento son "internas en interacción con el mundo externo, pero su énfasis teórico recae sobre los sucesos internos" (Papert; 1981, p. 186). No obstante, Papert marca una diferencia con Piaget en lo referente a la aplicación de esta teoría a la educación, afirmando que

mi perspectiva es más intervencionista. Mi meta es la educación, no la simple comprensión. De manera que en mi propia reflexión he colocado mayor énfasis en dos dimensiones implícitas pero no elaboradas en la propia obra de Piaget: el interés en las estructuras intelectuales que podrían desarrollarse, en oposición a aquellas que realmente se desarrollan actualmente en el niño, y el diseño de ambientes de aprendizaje que estén en consonancia con ellas (Papert; 1981, p. 186).

En la teoría de Piaget se está frente a un tipo de innatismo de las ideas, el ser humano está dotado de unas cualidades innatas (o instintos), no se trata de la tabla raza de Hume. Visto solamente este punto, el niño se convierte en un sujeto epistemológico; para Papert es "bueno para los niños ser epistemólogos" (Papert; 1981, p. 43), pues dichos instintos le posibilitan reaccionar con respecto a la realidad e ir produciendo paulatinamente esquemas mentales que a su vez crearán las diferentes estructuras mentales, distinguiendo las cuatro conocidas etapas: simbólica, intuitiva, concreta y formal (Piaget, 1967, p. 138Volumen 2, Número 1, Año 200217 
161). La realidad y el sujeto se construyen gracias a la actividad que media entre ambos. Según Devandas (1985, p. 129), el aporte más significativo de Papert a la epistemología reside en que la relación sujeto-objeto es eminentemente humana, en Papert, "el elemento relacional no será estrictamente humano, sino una máquina" (Devandas, 1985, p. 132). De esta manera, la teoría incorpora los factores tecnológicos contemporáneos.

Papert hace algunas modificaciones a la teoría de Piaget. Esos cambios son la introducción del afecto y el juego como elementos importantes en el proceso de aprendizaje. El niño aprende jugando y sobre todo, si sus juguetes son los queridos. Segundo, la posibilidad de transformar el orden de las etapas resumiéndolas en dos: las operaciones concretas y formal. Papert asevera que a Piaget se le olvidó este elemento tan importante (Papert; 1981, p. 12), es decir, Papert pretende hacer que el niño piense a edades tempranas de manera formal (Papert; 1981, p. 19). Esto en razón de una adecuación a los requerimientos y exigencias del ritmo de vida la sociedad contemporánea: brevedad, rendimiento, eficacia y productividad. En Piaget, los cambios están en función de la edad del niño, mientras que para Papert están en función de la cultura y se parte del supuesto de que el sistema cultural es la causa fundamental en el atraso mental. Así, la cultura computacional provee los materiales, situaciones y modos de pensar para que el niño a temprana edad resuelva problemas en los que se requiere tener un dominio claro de la combinación o de la conservación en términos piagetianos (Papert; 1981, p. 20).

Siguiendo los argumentos de Devandas, puede apreciarse que: la cultura precomputacional ciertamente provee también al niño de pensamiento sistemático. $\mathrm{Y}$, desde luego, esto se debe a la distorsión que en estas culturas se ha creado entre los estudios de ciencias que requieren y suponen la matemática y los que no (Devandas, 1985, p. 134)

Según el enfoque del optimismo ilustrado, las culturas anti-matemática crean a la vez un rechazo hacia la ciencia, ello dificulta también la resolución de problemas que requieren un pensamiento formal. Para esta perspectiva, el círculo vicioso que se genera de rechazo del pensamiento formal sólo puede romperse volcando los recursos para introducir las computadoras; así, basta con la introducción de la tecnología para producir el "desarrollo", aunque sea un proceso lento. 
Para esta perspectiva optimista ilustrada, la computadora es la tecnología salvadora (en este caso del modelo tradicional educativo y de las culturas anti-matemáticas) porque reúne dos características: (a) puede ser programada, (b) es sustentada por poderosas ideas matemáticas. El niño llegará gradualmente, de acuerdo con la adquisición de conocimientos, a convertirse en programador de la máquina. La adquisición de conocimiento es proporcional al grado de desarrollo mental del niño. Esto suena más a la forma tradicional del conocimiento. Con la diferencia de que dicho desarrollo se realiza a partir del papel del error y la depuración, en el proceso de autoaprendizaje. Una idea importante de Papert es la propuesta de respetar los diferentes estilos cognitivos.

Por último, la informática educativa constituye una herramienta importante para los procesos de aprendizaje de los niños y los adolescentes. Esta herramienta no es exclusiva, esto es, no representa la única vía por la cual los niños pueden llegar a "aprender a aprender". En este sentido, desarrollar destrezas de razonamiento en las personas menores de edad podría realizarse por medio de las clases de lógica, siempre y cuando estas respondan a las necesidades de los estudiantes, o por medio de la filosofía para niños. Estas didácticas pueden ser complementadas con las posibilidades que ofrecen las computadoras. Además, esas capacidades de aprender de manera agradable y no autoritaria no será posible si el sistema educativo no logra un cambio significativo en su sistema de enseñanza. Este cambio tiene que ver con las actitudes positivas que asuman los profesores y maestros en ese proceso. Muchos de ellos dependen de las políticas y estímulos que los gobiernos ofrezcan para que se produzcan las modificaciones. Sin embargo, debido a la educación masiva que parece ser inevitable por el crecimiento demográfico, da la impresión de que es imposible lograr una educación más personalizada, pero no por ello se debe descartar la posibilidad de hallar algún mecanismo que lo permita.

\footnotetext{
${ }^{10}$ Nótese un predominio de los enfoques lineales de la tecnología, como los de Hugo Padilla o las ideas de desarrollo lineal como las de Rostow.
} 


\section{Referencias}

Badilla Saxe, Elena; (1998). Reflexiones sobre informática educativa. Praxis, 51: 59-92.

Bellini, Luzia María, (1998). La escuela y el lenguaje Logo: el dilema del doble paradigma. Praxis, 51: 205-212.

Cabero, Julio. (2001). Tecnología educativa. Diseño y utilización de medios en la enseñanza. Barcelona: Paidós.

Camacho, Luis. (1985). Las objeciones de John Searle a la noción de inteligencia artificial y respuesta de D. Hofstadter. Praxis, 35-36: 187-194, Julio-Diciembre.

(1993). Algunas dudas sobre la revolución de la computación y la así llamada inteligencia artificial. Ciencia, tecnología en el subdesarrollo. Cartago: C.R.: Editorial Tecnológica de Costa Rica.

Chelque, José. (1999). Logo en los noventa una propuesta en crisis. Praxis, 51: 197-204.

Devandas, Carlos. (1985). Epistemología y aprendizaje por computadora. Praxis, 35-36: 125-126, Julio-Diciembre

Georgoudis, Dianelos. (1985). Computación y Educación. Praxis, 35-36: 101-119, JulioDiciembre.

Herra, Rafael Ángel. (1985). Apuntes sobre la ilusión cibernética. Praxis, 35-36: 181-186, Julio-Diciembre.

Huff, James; Salas, Carlos. (1985). Alfabetización en computadoras. Praxis, 35-36: 139147, Julio-Diciembre.

Misky, Marvin. (2000). La mente artificial. Newton, 27: 14-23, Julio.

Mitcham, Carl. (1989). ¿Qué es la filosofía de la tecnología? Barcelona: Editorial Anthropos.

Negroponte, Nicholas. (1995). Ser digital. Buenos Aires: Atlántida.

Papert, Syemort. (1981). Desafío de la mente: computadoras y educación. $5^{\mathrm{a}}$ ed., Buenos Aires: Ediciones Galápago.

. (1993). La máquina de los niños: replantearse la educación ella era de los ordenadores. Barcelona: Ediciones Paidós.

(1997). La Familia conectada. Argentina: Emecé.

Piaget, Jean. (1967). La psicología de la inteligencia. Barcelona: Crítica, 1999.

(1970). Las explicaciones causales. Barcelona: Barra, 1973. 
. (1973). Estudios de epistemología genética. Buenos Aires: Emecé, . . (1975). La equilibración de las estructuras cognitivas. Madrid: Siglo XXI.

Pozo, Juan Ignacio. (1997). Teorías cognitivas del aprendizaje. $5^{\mathrm{a}}$ ed., Madrid: Ediciones Morata.

Rifkin, Jeremy; (1994). El fin del trabajo: nuevas tecnologías contra puestos de trabajo: el nacimiento de una nueva era, Barcelona: Paidós.

Reparaz, Charo, y otros. (2000). Integración curricular de las nuevas tecnologías. Barcelona: Ariel.

Sánchez Illabaca, Jaime. (1992). Informática educativa. 2a ed., Santiago de Chile: Editorial Universitaria.

Saxe, Eduardo. (1998). La crisis neoliberal y el constructivismo racional en la educación costarricense. Praxis, 51: 17-47.

Seas, Yenny; Castro, Jorge; Corrales, Maricruz. (1999). Informática educativa. Aplicando escenarios para el aprendizaje. San José, C.R.: EUNED. 